\title{
Epistaxis in Children: Aetiology, Management and Outcome
}

\author{
SYED HASAN IMAM AL-MASUM ${ }^{1}$, ALI JACOB ARSALAN² ${ }^{2}$, DILRUBA BEGUM ${ }^{3}$
}

\begin{abstract}
Background: Although epistaxis is a common problem in preschool and school children, it is often considered as trivial and overlooked. As a result recurrent epistaxis sometimes may occur which, in some cases, may even be fatal.
\end{abstract}

Objective: The present study was, undertaken to determine the etiological profile and outcome of treatment of epistaxis in children.

\begin{abstract}
Methods: This cross sectional study was conducted in Dhaka Shishu Hospital, Dhaka from January 2012 to December 2013. In cases of mild bleeding and stable patient, detailed history was noted along with management of the bleeding. In case of severe bleeding, history was taken after the bleeding was controlled. The diagnosis of epistaxis was based on clinical history, physical findings, laboratory investigations with examination of the nose and nasopharynx. All patients were treated conservatively initially and chemical cauterization of the specific area was considered only when conservative means failed to control the epistaxis.
\end{abstract}

Results: Total 50 cases of epistoxis were enrolled. Nearly half (46\%) of the patients were 3-4 years old and the rest 54\% 5-6 years old with mean age being $4.6 \pm 0.9$ years. Majority (68\%) of the patients was male with male to female ratio being 2:1. Over twothirds (68\%) of the patients were from lower middle class. All patients' had anterior nasal bleeding. Seventy percent had acute epistaxis and the rest (30\%) had recurrent epistaxis. Nearly half (46\%) of the patients had inflammatory diseases, $28 \%$ had upper respiratory tract infections (URI), 16\% had history of nasal trauma, 12\% allergic rhinitis and very few patients (2\%) had idiopathic causes. Eight percent patients developed shock and were resuscitated along with management of bleeding. Over one-quarter (28\%) of the patients developed recurrent epistaxis and only $6 \%$ had facial oedema. All patients recovered uneventfully.

Conclusion: The study concluded that the common causes of epistaxis in children in our country is inflammatory and infectious diseases of nose and upper respiratory tract and most of the epistaxis could be managed with conservative treatment alone.

Key words: Epistaxis, causes, risk factors.

\section{Introduction}

Epistaxis or nasal bleeding is a problem frequently encountered in general practice and may present as an emergency, as a chronic problem of recurrent bleeds or may be a symptom of a generalized disorder. ${ }^{1}$ It is recognized as one of the most common

1. Associate Professor, ENT Department, Dhaka Shishu Hospital, Sher-e-Bangla Nagar, Dhaka.

2. Resident Medical Officer, ENT Department, Dhaka Shishu Hospital, Sher-e-Bangla Nagar, Dhaka.

3. Associate Professor, Department of Physiology, Dhaka. Medical College, Dhaka.

Correspondence: Dr. Syed Hasan Imam Al-Masum,

E-mail: shmasum_ent@yahoo.com otorhinolaryngological emergencies worldwide, particularly in children, and presents a challenge in resource-poor centres where facilities for caring of these patients are limited. ${ }^{2}$ It cannot only affect the hemodynamic stability but may cause great anxiety to their parents and relatives. It is estimated to occur in $60 \%$ of children worldwide during their lifetime, and approximately $6 \%$ of those with nosebleeds seek medical treatment. ${ }^{1-4}$ The prevalence is increased in children less than 10 years old. Generally, boys are slightly more affected than girls. ${ }^{1,4,5}$

Epistaxis is commonly divided into anterior and posterior epistaxis, depending on the site of origin. ${ }^{5}$ 
Anterior nosebleeds arise from damage to Kiesselbach's plexus on the lower portion of the anterior nasal septum, known as the Little's area, whereas posterior nosebleeds arise from damage to the posterior nasal septal artery. ${ }^{4,6}$ Anterior epistaxis is far more common than posterior epistaxis, accounting for more than $80 \%$ of cases. ${ }^{4,6,7}$

The aetiology of epistaxis can be broadly divided into the local or systemic causes, although even this distinction is difficult to make and the term "Idiopathic Epistaxis" is ultimately used in about $80-90 \%$ of the cases. ${ }^{4,8}$ The etiological profile of epistaxis has been reported to vary with age and anatomical location. ${ }^{4-8}$ Traumatic epistaxis is more common in younger individuals and is most often due to digital trauma, facial injury, or a foreign body in the nasal cavity. ${ }^{6-8}$ Non-traumatic epistaxis is rare in children and may due to systemic diseases and environmental factors (temperature, humidity, altitude) ${ }^{7,8}$ Epistaxis that occurs in children younger than 10 years usually is mild and originates in the anterior nose. ${ }^{9}$

The treatment of epistaxis requires a systematic and methodical approach, and options vary according to the cause, location, and severity of the hemorrhage. $4,6,7,9$ Both conservative and surgical treatment modalities have been used in the treatment of epistaxis. ${ }^{1,6}$ However, their outcome has never been evaluated in our setting partly because of paucity of local data. As most of the underlying causes of epistaxis are preventable. 8,9 a clearer understanding of the causes, treatment and outcome of these patients is essential for establishment of preventive strategies as well as treatment guidelines. ${ }^{2,7,8}$ This study was,therefore, conducted to identify the etiological profile and to determine the outcome of treatment of epistaxis in children.

\section{Materials and Methods}

This descriptive cross-sectional study was conducted on children who presented with nasal bleeding (epistaxis) at Dhaka Shisu Hospital, Sher-e-Bangla Nagar, Dhaka betweenJanuary 2012 to December 2013. In cases of mild bleeding and stable patient, detailed history was noted along with management of the bleeding. In case of severe bleeding, history was takenafter the bleeding was controlled. If there were signs of excessive blood loss and/or patient was in a state ofshock, steps were taken to stabilize the patient simultaneously with control of epistaxis. After resuscitation with fluid and electrolyes and silver nitrate cauterization of the Little's area supported by oral antibiotics, antihistamine and topical antibiotics, all patients underwent a detailed history taking and a thorough examination (systemic and local) with special emphasis to identify the site of bleeding.

The patients were subjected to investigations of hematological parameters. Blood samples were taken and sent for baseline haemoglobin estimation, bleeding time, clotting time, platelet count and blood grouping and cross matching when indicated. The diagnosis of epistaxis was based on clinical history, physical findings, laboratory investigations with examination of the nose and nasopharynx. All patients were treated conservatively initially and chemical cauterization of the Little's area with silver nitrate was done only when conservative means failed to control the epistaxis.

Conservative (non-surgical) treatment included anterior nasal packing and/or pressure over bleeding area with thumb and index finger for 5-6 minutes. Arterial ligation and endovascular embolization were not performed as there were no patients with intractable epistaxis. Successful treatment was defined as no recurrent epistaxis following pack removal or no readmission of children (who were admitted at in-patient with epistaxis) within 24 hours of hospital discharge. The data were collected using a pre-tested, structured questionnaire containing the variables of interest like demographics, cause of epistaxis, location of bleeding sites, management modalities, need for blood transfusion, length of hospital stay, complications and mortality. Collected data were analysed using Statistical Package for Social Sciences (SPSS), version 17.0. The mean and standard deviation for continuous variables and frequency with corresponding percentages for categorical variables were computed. Total 50 cases of epistaxis was enrolled.

\section{Results}

Total 50 cases of epiistaxis were enrolled. Nearly half $(46 \%)$ of the patients were 3-4 years old and the rest $54 \% 5-6$ years old. The mean age of the patients was $4.6 \pm 0.9$ years (range: $3-6$ years). Majority (68\%) of the children was male with male to female ratio being $2: 1$. Over two-thirds (68\%) of the patients were from lower middle class followed by poor (22\%) and middle class $(10 \%)($ Table-I).

Gross features of epistaxis show that all patients' had anterior nasal bleeding. Seventy percent had acute epistaxis and the rest $30 \%$ recurrent epistaxis 
(Table II). Nearly half $(46 \%)$ of the patients had inflammatory diseases, $28 \%$ had upper respiratory tract infections (URI) (Viral/Bacterial), 16\% had history nasal trauma, $12 \%$ allergic rhinitis and very few patients (2\%) had idiopathic causes (Table III). A few (8\%) patients developed shock and were resuscitated along with management of bleeding.More than onequarter $(28 \%)$ of the patients developed recurrent epistaxis and only $6 \%$ developed facial oedema (Table IV). Majority ( $88 \%$ ) of the patients was left alone with medical advice and observed and $12 \%$ needed anterior nasal packing. All patients recovered uneventfully (Table V).

Table-I

Distribution of children by their demographic characteristics $(n=50)$

\begin{tabular}{lcc}
\hline $\begin{array}{l}\text { Demographic } \\
\text { characteristics* }\end{array}$ & Frequency & Percentage \\
\hline Age $^{*}$ (years) & 23 & 46.0 \\
$5-6$ & 27 & 54.0 \\
$3-4$ & & \\
Sex & 34 & 68.0 \\
Male & 16 & 32.0 \\
Female & & \\
Socioeconomic status & 11 & 22.0 \\
Poor & 34 & 68.0 \\
Lower middle class & 5 & 10.0 \\
Middle class &
\end{tabular}

${ }^{*}$ Mean age $=4.6 \pm 0.9 ;$ range $=3-6$ years .

Table-II

Distribution of patients by their Gross features of epistaxis $(n=50)$

\begin{tabular}{lll}
\hline Gross features of & Frequency & Percentage \\
epistaxis & & \\
\hline
\end{tabular}

Area of bleeding

Anterior bleeding

$50 \quad 100.0$

Nature of epistaxis

\begin{tabular}{lll} 
Acute (single episode) & 35 & 70.0 \\
Recurrent & 15 & 30.0 \\
\hline
\end{tabular}

Table-III

Distribution of patients by their causes of epistaxis $(n=50)$

\begin{tabular}{lcc}
\hline Factors/causes & Frequency & Percentage \\
\hline Inflammatory diseases & 23 & 46.0 \\
URI (Viral/Bacterial) & 14 & 28.0 \\
Trauma & 6 & 12.0 \\
Allergic rhinitis & 4 & 8.0 \\
Thrombocytopenic purpura & 1 & 2.0 \\
Leukemia & 1 & 2.0 \\
Idiopathic & 1 & 2.0 \\
\hline
\end{tabular}

Table-IV

Distribution of patients by their complications developed $\left(n=50^{*}\right)$

\begin{tabular}{lcc}
\hline Complications developed & Frequency & Percentage \\
\hline Shock & 4 & 8.0 \\
Recurrent epistaxis & 14 & 28.0 \\
Facial oedema & 3 & 6.0 \\
\hline
\end{tabular}

*Total will not correspond to $100 \%$.

Table-V

Distribution of patients by management given $(n=50)$

\begin{tabular}{lcc}
\hline Management & Frequency & Percentage \\
\hline Observation alone & 44 & 88.0 \\
Anterior nasal packing & 6 & 12.0 \\
$5 \%$ Baby saline $(0.225 \mathrm{NaCl})$ & 03 & 6.0 \\
Blood transfusion needed & 01 & 2.0 \\
Mortality & 00 & 0.0 \\
\hline
\end{tabular}

Table-VI

Distribution of patients by their outcome $(n=50)$

\begin{tabular}{lcc}
\hline Outcome & Frequency & Mean \pm SD \\
\hline Good recovery $(n=50)$ & $50(100.0)$ & - \\
Hospital stay (days) & - & $3.1 \pm 0.6$ \\
\hline
\end{tabular}

\section{Discussion}

The present study intended to find the determinants and outcome of management included a total of 50 children of average 4.6 years old. Male female ratio was 2:1. Ninety percent of the children belonged lower middle and poor class family. All patients in our study exhibited anterior nasal bleeding which corresponds well with findings of Viljoen et al ${ }^{10}$ who demonstrated that $90 \%$ of the epistaxis in children originates from Little's area in the anterior part of the nose, often being either idiopathic or the result of trauma. Epistaxis that occurs in children younger than 10 years usually is mild and originates in the anteriornose. ${ }^{9}$ 
The etiological profile of epistaxis has been reported to vary with age and anatomical location. ${ }^{11-13}$ But in our setting the most common aetiological cause of epistaxis was observed to be inflammatory $(46 \%)$ and upper respiratory tract infections $(28 \%)$. Of the rare causes, $1(2 \%)$ had leukemia and another $1(2 \%)$ had thrombocypenic purpura.

Although bleeding may occur spontaneously, it often results from forceful nose blowing and sneezing which increases arterial and venous pressure in the vascularised nasal septum, which usually accompanies allergic rhinitis, viral/bacterialURIs and trauma/sepsis secondary to foreign bodies. Posterior epistaxis is uncommon in children and no case was found in our series. Posterior epistaxis is usually the result of bleeding disorders, inflammatory disorders or neoplasms and occurs in individuals older than 50 years. ${ }^{11}$ Persistent or recurrent epistaxis should raise the suspicion of bleeding disorders or neoplasms, necessitating further investigation.

In terms of complications, $4(8 \%)$ patients at presentation were in a state of shock and were resuscitated with fluid and electrolytes. One of them however, required blood transfusion as well. Of the 50 patients $14(28 \%)$ had recurrent epistaxis and $3(6 \%)$ developed facial oedema. However, all complications were successfully managed and recovered uneventfully. In our setting majority of the cases were managed with conservative treatment which included anterior nasal packing and/or firm pressure over bleeding area with thumb and index finger. However, $3(6 \%)$ cases required to be resuscitated with saline and $1(2 \%)$ case required blood transfusion. The treatment of epistaxis requires a systematic and methodical approach, and options vary according to the cause, location, and severity of the hemorrhage..$^{9,11,13}$ Both conservative and surgical treatment modalities have been used in the treatment of epistaxis. ${ }^{1,6}$ Chemical cauterization of the specific area is considered only when conservative modality of treatment failed to control the epistaxis.

Taking a proper history and performing a thorough systemic examination are imperative to rule out the possibility of the bleeding being the result of systemic disease or bleeding disorders (petechiae, purpura, ecchymosis, hepatosplenomegaly), which will necessitate further investigations. A positive family history and prolonged bleeding and clotting time are useful predictive criteria to detect family history of bleeding disorders. Resuscitation is seldom required in children and always signifies more complex cases.

\section{Conclusion}

The study concluded that the common causes of epistaxis in children in our country is inflammatory and infectious diseases of nose and upper respiratory tract and most of the epistaxis could be managed with conservative treatment alone. However, largescale study is needed to validate the findings of the present study.

\section{References}

1. Pond F, Sizeland A. Epistaxis. Strategies for management. Aust Fam Physician 2000; 29: 933-38.

2. Akinpelu OV, Amusa YB, Eziyi JA, Nwawolo CC. $A$ retrospective analysis of aetiology and management of epistaxis in a south-western Nigerian teaching hospital. West Afr J Med 2009; 28:165-68.

3. Yueng-Hsiang $\mathrm{C}$, Jih-Chin L: Unilateral Epistaxis. N Eng J Med 2009; 361:14.

4. Ciaran $\mathrm{SH}, \mathrm{O}$ wain $\mathrm{H}$. Update on management of epistaxis. The West London Medical Journal 2009;1:33-41.

5. Walker TWM, Macfarlane TV, McGarry GW. The epidemiology and chronobiology of epistaxis: an investigation of Scottish hospital admissions 1995-2004. Clin Otolaryngol 2007;32:361-65.

14. Pope LER, Hobbs CGL. Epistaxis: an update on current management. Postgrad Med J 2005;81:309-14.

7. Nash CM, Field SMB. Epidemiology of Epistaxis in a Canadian Emergency Department. Israeli J Emerg Med 2008;8:24-28.

8. Pallin DJ, Chng Y, McKay MP, Emond JA, Pelletier AJ, Camargo CA. Epidemiology of epistaxis in US emergency departments, 1992 to 2001. Ann Emerg Med 2005, 46:77-81.

9. Bernius M, Perlin D. Pediatric ear, nose, and throat emergencies. Pediatr Clin North Am 2006; 53:195.

10. Viljoen J. Epistaxis in children: approach and management. CME 2003;21:664-65.

11. Ciaran $\mathrm{SH}$, Owain $\mathrm{H}$. Update on management of epistaxis. The West London Med J 2009;1: 33-41.

12. Walker TWM, Macfarlane TV, McGarry GW. The epidemiology and chronobiology of epistaxis: an investigation of Scottish hospital admissions 1995-2004. Clin Otolaryngol 2007;32:361-65.

13. Nash CM, Field SMB: Epidemiology of Epistaxis in a Canadian Emergency Department. Israeli J Emerg Med 2008;8:24-28. 